ARTICLE

\title{
Heterogeneity and clinical significance of ESR1 mutations in ER-positive metastatic breast cancer patients receiving fulvestrant
}

Jill M. Spoerke ${ }^{1, \star}$, Steven Gendreau ${ }^{1, \star}$, Kimberly Walter ${ }^{1, \star}$, Jiaheng Qiu ${ }^{1}$, Timothy R. Wilson ${ }^{1}$, Heidi Savage Junko Aimi ${ }^{1}$, Mika K. Derynck ${ }^{1}$, Meng Chen ${ }^{1, \dagger}$, Iris T. Chan ${ }^{1}$, Lukas C. Amler ${ }^{1}$, Garret M. Hampton', Stephen Johnston ${ }^{2}$, Ian Krop ${ }^{3}$, Peter Schmid ${ }^{4} \&$ Mark R. Lackner ${ }^{1}$

Mutations in ESR1 have been associated with resistance to aromatase inhibitor (AI) therapy in patients with ER + metastatic breast cancer. Little is known of the impact of these mutations in patients receiving selective oestrogen receptor degrader (SERD) therapy. In this study, hotspot mutations in ESR1 and PIK3CA from ctDNA were assayed in clinical trial samples from $\mathrm{ER}+$ metastatic breast cancer patients randomized either to the SERD fulvestrant or fulvestrant plus a pan-PI3K inhibitor. ESR1 mutations are present in $37 \%$ of baseline samples and are enriched in patients with luminal $A$ and PIK3CA-mutated tumours. ESR1 mutations are often polyclonal and longitudinal analysis shows distinct clones exhibiting divergent behaviour over time. ESR1 mutation allele frequency does not show a consistent pattern of increases during fulvestrant treatment, and progression-free survival is not different in patients with ESR1 mutations compared with wild-type patients. ESR1 mutations are not associated with clinical resistance to fulvestrant in this study.

\footnotetext{
${ }^{1}$ Genentech, Inc, South San Francisco, California 94080, USA. ${ }^{2}$ Royal Marsden NHS Foundation Trust, London SW3 6JJ, UK. ${ }^{3}$ Dana-Farber Cancer Institute, Boston, Massachusetts 02215, USA. ${ }^{4}$ Barts Cancer Institute, Queen Mary University London, London EC1M 6BQ, UK. * These authors contributed equally to this work. †Present address: Roche China Holding, Shanghai 201203 China. Correspondence and requests for materials should be addressed to M.R.L. (email: lackner.mark@gene.com).
} 
O estrogen receptor alpha $(E R \alpha)$, encoded by the ESR1 gene, is a member of the nuclear hormone receptor superfamily that is expressed in $\sim 70 \%$ of newly diagnosed breast cancers ${ }^{1}$. ER $\alpha$ and its cognate ligand oestrogen are the major drivers of tumour development and disease progression in luminal breast cancers, and agents that impair ER signalling in ER-positive breast cancers represent highly successful targeted therapies that are widely used in both early breast cancer, as well as the metastatic setting ${ }^{2}$. The selective oestrogen receptor modulator tamoxifen was approved in 1997 and still represents the standard of care for adjuvant treatment of early breast cancer ${ }^{3}$ especially in premenopausal patients, though aromatase inhibitors (AIs) that block biosynthesis of oestrogen are commonly incorporated into adjuvant treatment algorithms 4 and represent a first-line standard of care in advanced, metastatic breast cancer in postmenopausal patients ${ }^{5}$. While the SERD fulvestrant is similar to selective oestrogen receptor modulators in antagonizing ER transcriptional activity, fulvestrant also causes ER degradation and has proven to be just as effective as AIs in advanced breast cancer ${ }^{2,6}$.

Despite the effectiveness of the various anti-hormonal therapies, intrinsic and acquired resistance remains a persistent problem that limits the ultimate effectiveness of these treatments ${ }^{7}$. The issue is particularly acute in the ER + metastatic breast cancer (MBC) setting, where up to half of all patients show intrinsic resistance and do not benefit from therapy, and ultimately all ER + MBC patients develop acquired resistance and progress on anti-hormonal therapies ${ }^{5}$. Preclinical and clinical investigations have demonstrated that these resistant ER + breast cancers often retain $\mathrm{ER} \alpha$ expression and dependence on oestrogen receptor signalling. In clinical practice, continued ER dependence is observed with patients who have progressed on one form of endocrine therapy ${ }^{8}$ often remaining responsive to different endocrine therapies in subsequent lines of therapy ${ }^{9}$. The discovery of acquired mutations in ESR1 that confer ligandindependent and constitutive activation of $\mathrm{ER} \alpha$ revealed one potential mechanism of resistance to endocrine therapies. ESR1 mutations were originally reported in a small cohort of metastatic breast cancers in 1997 (ref. 10), and confirmed recently in several larger studies that utilized next-generation sequencing and suggested that such mutations are present in $\sim 20 \%$ of metastatic tissue samples but are generally not found in primary tumour samples ${ }^{11-13}$. The most frequently occurring ESR1 mutations are in the ligand-binding domain (LBD) of ER $\alpha$, generally clustering between amino acids 534-538, though mutations at other positions including S463 and E380 have also been described. Biochemical studies demonstrated that the Y537S mutation is constitutively active and results in full transcriptional activity in the absence or presence of oestrogen ${ }^{14}$, and subsequent reports demonstrated that other $\mathrm{ER} \alpha \mathrm{LBD}$ mutations similarly confer constitutive, ligand-independent activation ${ }^{12,13,15}$. Functional in vitro studies, along with the observation that mutations were found in metastatic tissue collected from patients who had undergone therapy with AIs, suggest a model wherein these mutations have an important role in acquired resistance to oestrogen deprivation mediated by $\mathrm{AIs}^{16}$.

An important unresolved question is whether $\mathrm{ER} \alpha \mathrm{LBD}$ mutations retain sensitivity to other endocrine agents, particularly SERDs such as fulvestrant that cause $\mathrm{ER} \alpha$ degradation. In vitro findings have suggested that mutant ER $\alpha$ can still bind and be inhibited by fulvestrant, but that higher doses are required to inhibit mutant $\mathrm{ER} \alpha$ compared with wild-type (WT) $\mathrm{ER} \alpha^{12,13}$. Little is known as to how these mutations affect clinical outcomes in patients receiving endocrine therapies, and whether SERD treatment can achieve high enough clinical exposures to effectively degrade mutant ER $\alpha$. The FERGI study (GDC4950g,
NCT01437566) is a randomized phase 2 study that compared the pan-PI3K inhibitor pictilisib plus fulvestrant with placebo plus fulvestrant in patients with $\mathrm{ER}+\mathrm{MBC}$ who had received prior $\mathrm{AI}$ therapy ${ }^{17}$. As such, the FERGI study represents an excellent setting to analyse the prevalence of ESR1 mutations in MBC patients that failed prior $\mathrm{AI}$, as well as to explore the impact of these mutations on the therapeutic benefit of fulvestrant in these patients. In this study, baseline and on-treatment ESR1 and PIK3CA mutation status was assessed in plasma samples from patients enrolled in the FERGI study. Several recent studies have shown the feasibility of detecting ESR1 mutations in circulating tumour DNA (ctDNA) and that the mutation status in plasma DNA can more accurately reflect the extent of disease heterogeneity than the analysis of tissue from a single metastatic site $\mathrm{e}^{18-20}$. In addition, ctDNA analyses offer the possibility of non-invasive monitoring of tumour mutation status over time and treatment, an approach that has been used successfully in the identification of circulating KRAS mutations in colorectal patients receiving anti-epidermal growth factor receptor (EGFR)-targeted therapies ${ }^{21}$, but which has not been well explored in the context of ESR1 mutations and endocrine therapies. The major findings reported herein are that ESR1 mutations are present in $\sim 40 \%$ of patients that have progressed on AI therapy and are numerically enriched in luminal A and PIK3CA-mutated tumours. Of the patients with a detectable ESR1 mutation, $40 \%$ harbour multiple ESR1 mutations, suggesting the convergent evolution of multiple AI-resistant clones. Serial monitoring of PIK3CA and ESR1 mutations in plasma from 71 patients suggests that RECIST responses are associated with decreases in allele frequency (AF) for both PIK3CA and ESR1, but decreases in on-treatment AF did not reliably predict response since patients with stable or progressive disease (SD/PD) in some cases show decreases. In contrast, increases in on-treatment AF are only seen in patients with best response of SD or PD. Most importantly, ESR1 mutations are not associated with differential progression-free survival (PFS) benefit in either the fulvestrant control arm or the experimental arm, suggesting that they may not be strongly associated with clinical resistance to SERD treatment.

\section{Results}

ESR1 and PIK3CA mutations in ctDNA baseline plasma samples. We used a sensitive and quantitative digital PCR assay to screen for 9 hotspot mutations in PIK3CA and 12 mutations in ESR1 in ctDNA (see Methods). Table 1 shows PIK3CA and ESR1 mutations as they relate to baseline clinical and demographic features, while Supplementary Table 1 shows the prevalence of PIK3CA and ESR1 in breast cancer subsets. PIK3CA mutations were detected in ctDNA from $39.7 \%(62 / 156)$ of patients from the unselected phase of the study (part 1), which enrolled patients irrespective of PIK3CA tissue status (Table 1). The majority of patients $(90.3 \%, 56 / 62)$ had only a single detectable PIK3CA mutation in ctDNA (Table 1). ESR1 mutations were detected in ctDNA from $37.3 \%(57 / 153)$ of patients at baseline and exhibited markedly more heterogeneity than PIK3CA mutations. Among patients with any detectable ESR1 mutation, 22.8\% (13/57) had two mutations and $17.5 \%(10 / 57)$ had greater than two mutations. Mutations resulting in the amino-acid substitutions D538G, Y537S and E380Q were the most common alterations, found in $54 \%, 33 \%$ and $26 \%$ of ESR1 mutant samples, respectively (Fig. 1a). In addition, the PIK3CA mutations had a higher median AF than ESR1 (3.6\% versus $0.45 \%$ ) and samples with mutations in both genes generally showed a higher AF for PIK3CA compared with ESR1 (Fig. 1b and Supplementary Fig. 1). Of the 11 ESR1 mutations we detected in ctDNA, transiently expressed ER $\alpha$ 


\begin{tabular}{|c|c|c|c|}
\hline \multicolumn{4}{|c|}{ Part 1: randomized GDC-0941 and placebo GDC-0941 patients } \\
\hline & $\begin{array}{l}\text { ESR1 wild type, } 96 \\
(62.7 \%)\end{array}$ & $\begin{array}{c}\text { ESR1 mutation, } 57 \\
(37.3 \%)\end{array}$ & $\begin{array}{l}\text { Total, } 153 \\
(100 \%)\end{array}$ \\
\hline \multicolumn{4}{|c|}{ ESR1 mutation number } \\
\hline 0 & $96(100 \%)$ & 0 & $96(62.7 \%)$ \\
\hline 1 & 0 & $34(59.6 \%)$ & $34(22.2 \%)$ \\
\hline 2 & 0 & $13(22.8 \%)$ & $13(8.5 \%)$ \\
\hline $3+$ & 0 & $10(17.5 \%)$ & $10(6.5 \%)$ \\
\hline \multicolumn{4}{|c|}{ PIK3CA status ctDNA } \\
\hline Wild type & $68(70.8 \%)$ & $23(40.4 \%)$ & $91(59.5 \%)$ \\
\hline Mutation & $28(29.2 \%)$ & $34(59.6 \%)$ & $62(40.5 \%)$ \\
\hline \multicolumn{4}{|c|}{ Luminal status } \\
\hline Luminal $\mathrm{A}$ & $58(60.4 \%)$ & $41(71.9 \%)$ & $99(64.7 \%)$ \\
\hline Luminal B & $30(31.3 \%)$ & $14(24.6 \%)$ & $44(28.8 \%)$ \\
\hline Other & $8(8.3 \%)$ & $2(3.5 \%)$ & $10(6.5 \%)$ \\
\hline \multicolumn{4}{|c|}{ Visceral disease } \\
\hline Yes & $45(46.9 \%)$ & $35(61.4 \%)$ & $80(52.3 \%)$ \\
\hline No & $51(53.1 \%)$ & $22(38.6 \%)$ & $73(47.7 \%)$ \\
\hline \multicolumn{4}{|c|}{ Resistance to prior $\mathrm{Al}$} \\
\hline Primary & $42(43.8 \%)$ & $15(26.3 \%)$ & $57(37.3 \%)$ \\
\hline Secondary & $54(56.3 \%)$ & $41(71.9 \%)$ & $95(62.1 \%)$ \\
\hline Unknown & 0 & $1(1.8 \%)$ & $1(0.7 \%)$ \\
\hline \multicolumn{4}{|c|}{ Number of metastatic sites } \\
\hline 0 & $5(5.2 \%)$ & $1(1.8 \%)$ & $6(3.9 \%)$ \\
\hline 1 & $37(38.5 \%)$ & $13(22.8 \%)$ & $50(32.7 \%)$ \\
\hline \multirow[t]{2}{*}{$2+$} & $54(56.3 \%)$ & $43(75.4 \%)$ & $97(63.4 \%)$ \\
\hline & $\begin{array}{c}\text { PIK3CA wild type, } \\
94(60.3 \%)\end{array}$ & $\begin{array}{c}\text { PIK3CA mutation, } \\
62(39.7 \%)\end{array}$ & $\begin{array}{l}\text { Total, } 156 \\
(100 \%) \\
\end{array}$ \\
\hline \multicolumn{4}{|c|}{ PIK3CA mutation number } \\
\hline 0 & $94(100 \%)$ & 0 & $94(60.3 \%)$ \\
\hline 1 & 0 & $56(90.3 \%)$ & $56(35.9 \%)$ \\
\hline $2+$ & 0 & $6(9.7 \%)$ & $6(3.8 \%)$ \\
\hline \multicolumn{4}{|c|}{ Luminal status } \\
\hline Luminal $\mathrm{A}$ & $56(59.6 \%)$ & $44(71.0 \%)$ & $100(64.1 \%)$ \\
\hline Luminal B & $28(29.8 \%)$ & $17(27.4 \%)$ & $45(28.8 \%)$ \\
\hline Other & $10(10.6 \%)$ & $1(1.6 \%)$ & $11(7.1 \%)$ \\
\hline
\end{tabular}

proteins encoded by the most commonly detected mutations generally showed the strongest ligand-independent transcriptional activity in vitro, with the exception of E380Q that did not show significantly increased ligand-independent activity relative to WT ER $\alpha$ (Supplementary Fig. 2). Overall PIK3CA mutations in ctDNA were $86 \%$ concordant with tissue PIK3CA status determined by quantitative PCR assay (77\% sensitivity and $93 \%$ specificity; Fig. 2a, Supplementary Table 2 and Supplementary Data 1). We also compared ESR1 mutation status between tissue and plasma for a subset of patients and examined the relationship between the type of tissue (primary tumour or metastatic lesion) and collection time relative to AI therapy in the metastatic setting (Fig. 2b). We did not find any ESR1 mutations in primary tumour tissue collected at diagnosis, consistent with previous reports. Moreover, we rarely found ESR1 mutations in tissue that was collected before AI therapy (3/81 patients), but did detect ESR1 mutations in tissue that was collected after progression on prior AI therapy in a much higher proportion of patients (12/21 patients). The most abundant ESR1 mutation detected in plasma (that is, highest AF) was almost always the mutation detected in
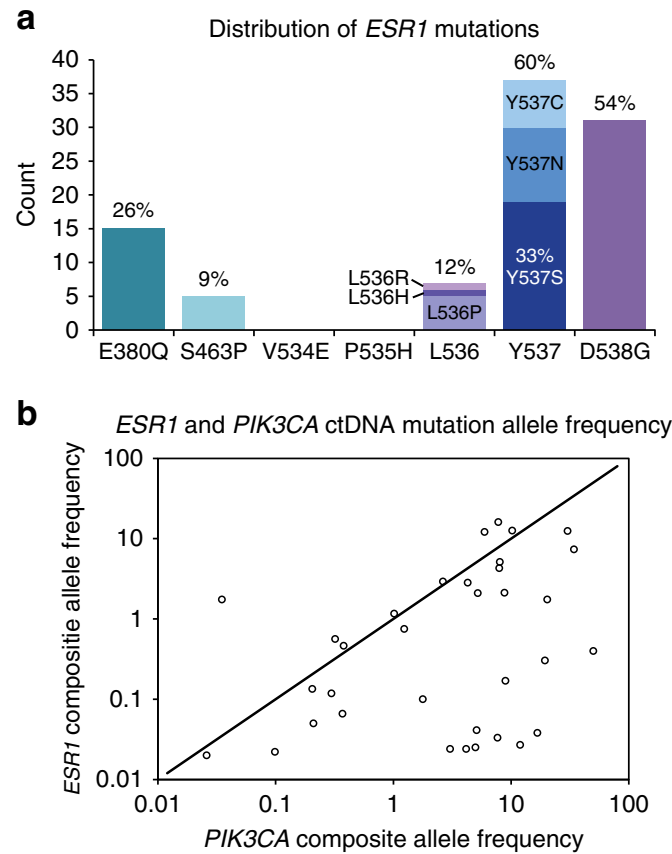

Figure 1 | Distribution of ESR1 mutations and AF. (a) Distribution of ESR1 mutations detected in ctDNA from 153 part 1 patients. The bars indicate the number of patients for which each mutation was detected, and the percentage is relative to the total number of patients with mutations detected (57). Since multiple mutations were detected in $40 \%$ of the patients, the sum of the percentages is $>100 \%$. P535H and L536Q were not detected in any of the part 1 samples, but each was detected once among the 54 samples from part 2. V534E was not detected in any part 1 or part 2 sample. (b) Scatter plot of ESR1 and PIK3CA ctDNA mutation allele frequency (AF) for patients where both mutations were detected $(n=34)$. The mutation AF sum is plotted for patients with multiple ESR1 or PIK3CA mutations.

tissue (Supplementary Table 3). The overall concordance of ESR1 tissue and ctDNA status was $31 \%$ when primary and metastatic samples are pooled together. The concordance between ESR1 status in ctDNA and metastatic tissue was $47 \%$, while concordance between ctDNA and primary tissue was 5\%. Concordance was $57 \%$ and $23 \%$ for patients whose tumour tissue was collected after or before the administration of an aromatase inhibitor, respectively (Supplementary Table 4).

Association of mutations with clinical features. We examined the relationship between plasma ESR1 mutation status and a range of baseline clinical and demographic features. Overall, ESR1 mutations occurred more frequently in patients who also harboured PIK3CA mutations, as $44.4 \%$ of patients with PIK3CA mutations detected in tissue also had a plasma ESR1 mutation compared with $30.3 \%$ of patients without a PIK3CA mutation (Supplementary Table 1). Plasma ESR1 mutations were also found in $44.4 \%$ of patients enrolled in part 2 of the FERGI study, which selected patients based on PIK3CA mutation tissue status (Supplementary Table 1, Supplementary Fig. 3 and Supplementary Data 1). The presence of an ESR1 mutation in the plasma also showed a trend towards enrichment in patients with luminal A status determined by PAM50 algorithm ${ }^{22}$, as $41.4 \%$ of patients with PAM50 defined luminal A tumours had ESR1 mutations compared with $31.8 \%$ of patients with luminal B tumours (Table 1 and Supplementary Table 1). A smaller 

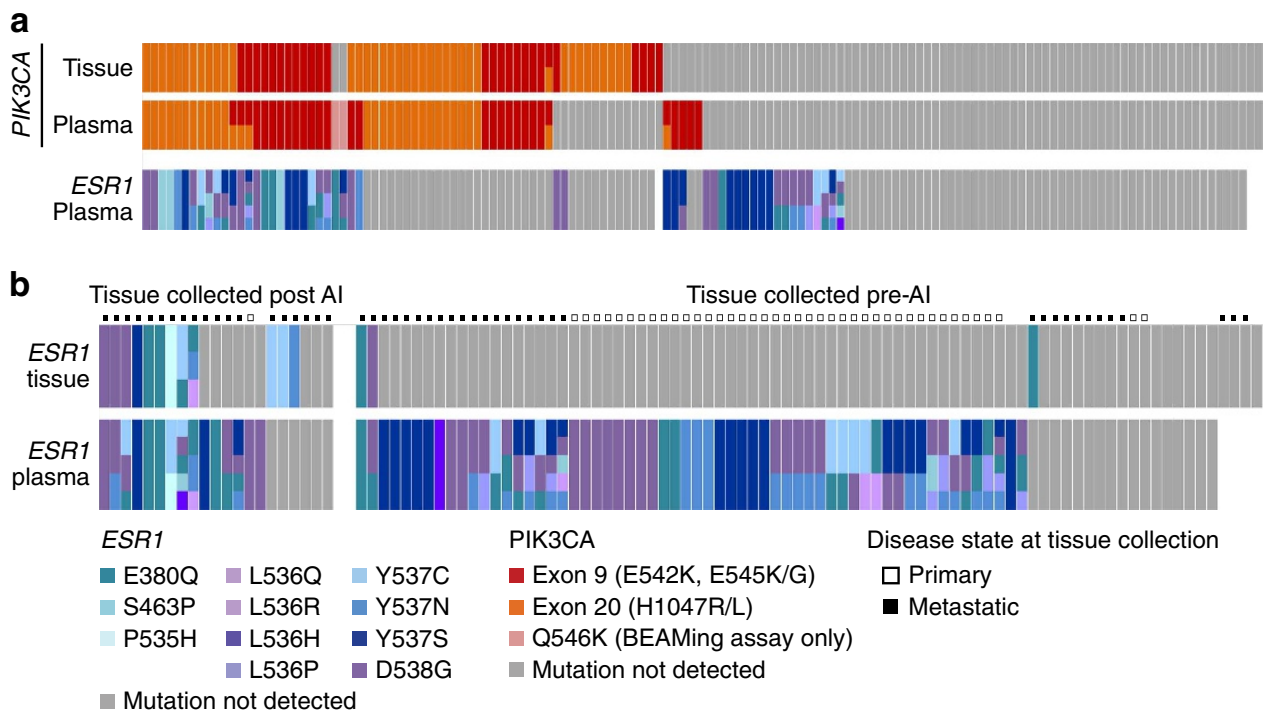

Figure 2 | PIK3CA and ESR1 mutation analysis of matched tissue and plasma samples. (a) PIK3CA and ESR1 mutation analysis of matched tumour tissue and plasma samples from 142 patients from part 1 of the clinical study. Mutations are coloured according to exon (PIK3CA) or amino acid (ESR1). Grey indicates mutations assessed were negative, and blank indicates data not available. (b) ESR1 tissue and matched plasma mutation status from 102 part 1 and part 2 patients. Patients whose tissue was collected after receiving Al therapy shown on the left (21), and those whose tissue was collected before Al on the right (81). Tissue collection at the time of primary or metastatic disease indicated with open or solid squares, respectively.

numerical increase was seen in patients with $\mathrm{PgR}+(38.5 \%)$ compared with $\mathrm{PgR}-(33.3 \%)$ tumours. Increases in plasma ESR1 mutation prevalence were also associated with several clinicopathologic parameters. Sixty-one per cent of patients with ESR1 mutations had visceral disease, whereas only $46.9 \%$ of ESR1 WT patients had visceral disease (Table 1). In addition, $71.9 \%$ of patients with ESR1 mutations had secondary resistance to prior AI therapy compared with $56.3 \%$ of ESR1 WT patients, and $75.4 \%$ of patients with ESR1 mutations had two or more metastatic sites compared with $56.3 \%$ of ESR1 WT patients. Other demographic and clinical parameters were generally balanced between patients with ESR1 mutations and ESR1 WT patients, including age, race, region, Eastern Cooperative Oncology Group (ECOG) score, baseline tumour burden and others (Supplementary Table 5). In similar analyses, PIK3CA mutations in ctDNA generally were not associated with particular demographic and clinical features (Supplementary Table 6), with the exception of luminal status (Table 1). Similar to ESR1, a slightly higher proportion of patients with luminal A tumours had PIK3CA mutations in ctDNA compared with patients with luminal B tumours $(44 \%, 44 / 100$ versus $38 \%, 17 / 45)$.

Association of baseline mutations with clinical outcome. The FERGI study was a randomized phase 2 study comparing the pan-PI3K inhibitor pictilisib plus fulvestrant with placebo plus fulvestrant in patients with ER + locally advanced or metastatic breast cancer, and so is an excellent setting to examine the association of ESR1 and PIK3CA mutations in ctDNA with clinical outcome to these agents. PIK3CA status determined by quantitative PCR assay on archival tumour tissue was not associated with enhanced clinical benefit from the addition of pictilisib to fulvestrant in the FERGI study ${ }^{17}$. Here we retrospectively analysed whether PIK3CA and ESR1 mutations detected in ctDNA were associated with differential benefit in either the control or the experimental arm of FERGI. Patients with a detectable plasma ESR1 mutation did not show differential PFS in either the fulvestrant or fulvestrant plus pictilisib arm (Fig. 3 and
Supplementary Fig. 4A). We also examined whether patients with more than one ESR1 mutation (Fig. 3a) or a higher than median AF (Fig. 3b) showed differential outcome in the fulvestrant control arm, based on previous reports suggesting higher AFs of $B R A F$ V600E mutations are associated with higher disease burden and shorter PFS to BRAF-targeted therapy ${ }^{23}$. Neither the presence of multiple ESR1 mutations nor higher ESR1 AFs were associated with a clear difference in risk of progression on fulvestrant (Fig. 3). Patients with a PIK3CA mutation in ctDNA had a PFS hazard ratio of 0.994 compared with PIK3CA WT patients in the fulvestrant control arm, and showed a PFS hazard ratio of 0.991 comparing PIK3CA WT patients in the fulvestrant plus pictilisib arm, with overlapping 95\% confidence intervals (Supplementary Fig. 4B).

Mutant AF changes and tumour response. Longitudinal plasma samples from 71 patients were used to look at changes in AF over the course of treatment. Of these, 60 patients had at least one ESR1 or PIK3CA mutation detected in at least one time point (Supplementary Fig. 5). Patients were binned based on their best clinical response irrespective of treatment, with complete or partial responses (CR/PR) defined by RECIST criteria, and stable disease (SD) or progressive disease (PD) as assessed by the investigator. Patients whose best response was $\mathrm{CR}$ or $\mathrm{PR}$ demonstrated robust decreases in ESR1 and PIK3CA AF post treatment and did not show any evidence of increases in AF for either ESR1 or PIK3CA at the time points monitored (Fig. 4a,b and Supplementary Fig. 6). In contrast, patients whose best response was $\mathrm{SD}$ or $\mathrm{PD}$ displayed more variable changes in AFs, with some mutations decreasing at cycle 2 but many others remaining unchanged or increasing at this time point. We also looked at whether ESR1 mutation AF showed reproducible changes in the fulvestrant control arm, which might be predicted if these alterations resulted in structural changes in the protein that impaired the ability of fulvestrant to bind to ER and promote degradation of mutant ER protein. In fact, we found that a substantial majority of ESR1 mutations in ctDNA showed decreases 
a

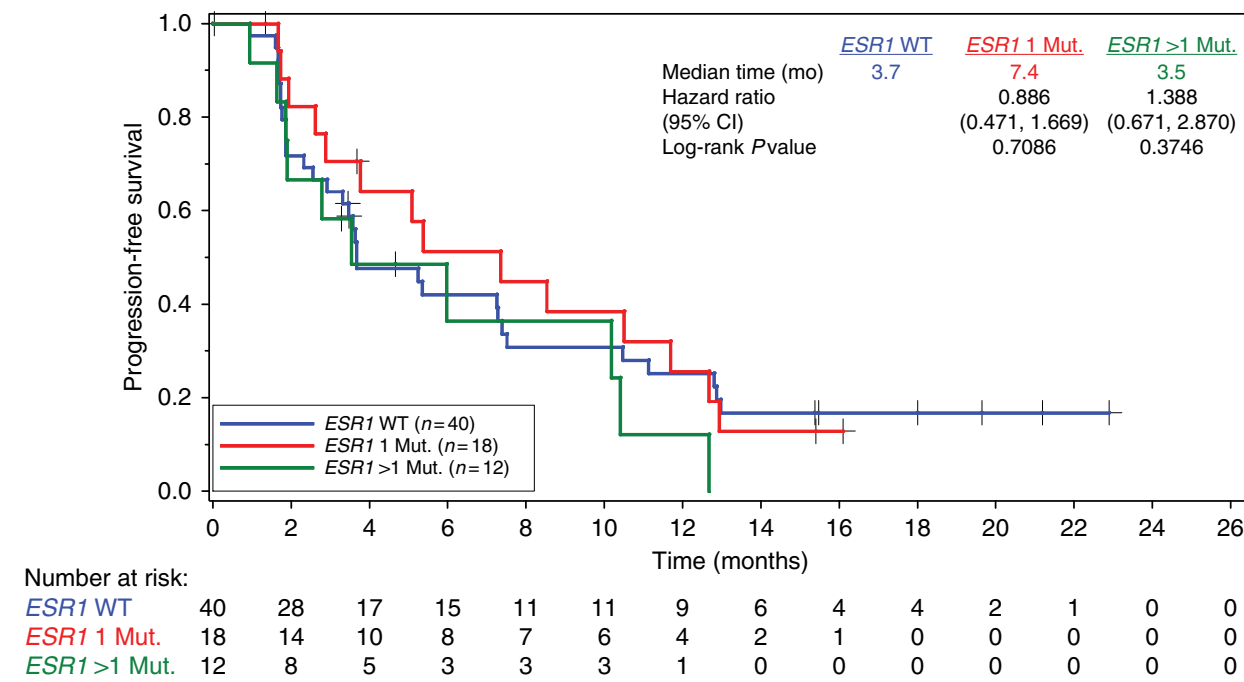

b

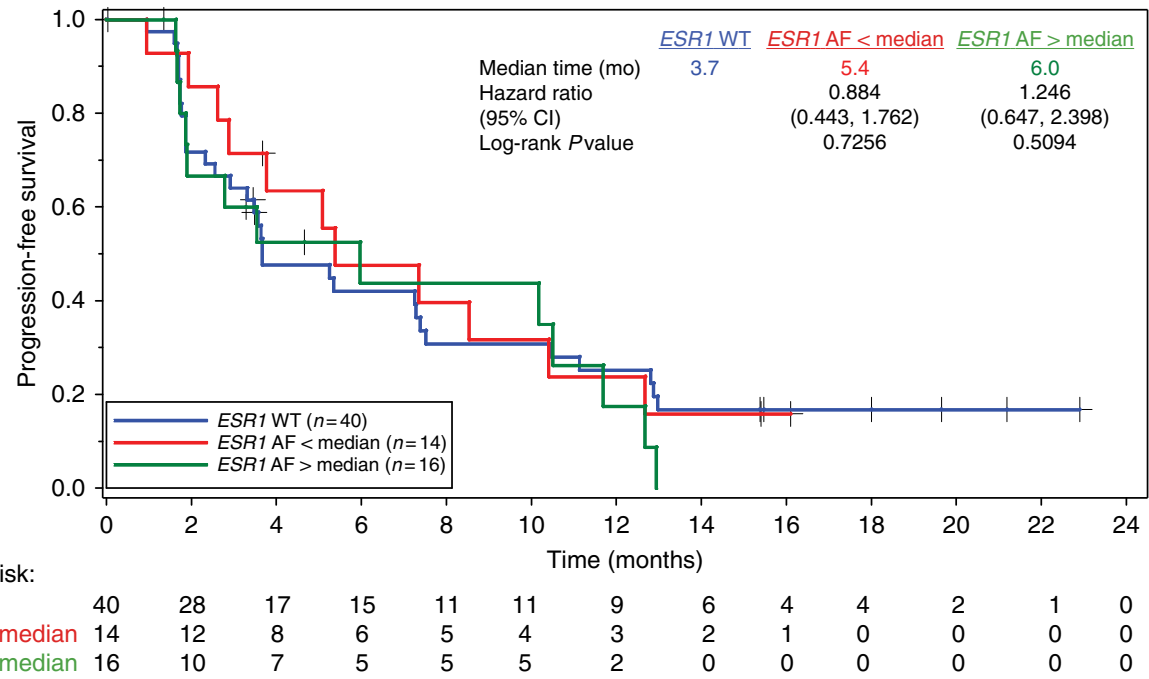

Figure 3 | Kaplan-Meier plots of PFS in the fulvestrant and placebo part 1 patients. (a) PFS of ESR1 mutation (Mut.) status divided by one (red) or greater than one (green) mutation, or WT (blue). (b) PFS by ESR1 mutation AF less than (red) or greater than (green) the median AF. Hazard ratios are relative to WT for both plots. mo, months.

in $\mathrm{AF}$ at the initial time point and most of these decreases persisted at later time points (Fig. 4a and Supplementary Fig. 6). We also did not detect any trend towards particular ESR1 hotspot mutations showing increases at any given time point (Supplementary Fig. 5), and did not detect the appearance of new ESR 1 mutations at later time points in the majority of patients. Specifically, of the 14 patients randomized to the fulvestrant control arm and on study for at least 140 days with no detectable ESR1 mutations at baseline, 10 continued to be free of detectable plasma ESR1 mutations. Two patients (1507 and 5801) showed the appearance of low-frequency ESR1 mutations at cycle 6, one at D538G $(0.12 \%)$ and the second with a D538G $(0.09 \%)$ and Y537N (0.12\%) mutation. Another two patients (1655 and 3501) had progressive disease and crossed over to the pictilisib plus fulvestrant arm and had detectable ESR1 mutations at cycle 2 of this regimen, one with E380Q (0.13\%) and the second with Y537C (0.08\%).

Intrapatient monitoring suggests clonal heterogeneity. Intrapatient patterns of AF changes over time were examined in all 60 patients with longitudinal data who had at least one detectable mutation. The complete data set is shown in Supplementary
Fig. 5, and we highlight several patients that show utility for monitoring differential response to therapy based on clonal heterogeneity (Fig. 5). Patient 2310 showed evidence of a PIK3CA mutation that started at relatively low AF markedly increased over treatment. A concomitant D538G mutation in ESR1 tracked well with the PIK3CA mutation over time and treatment. However, additional ESR1 mutations Y737S and Y737N showed divergent behaviour and did not increase over time. Patient 6951 showed evidence of a PIK3CA mutation that increased in AF over time, while concomitant ESR1 mutations either remained unchanged or showed a slight increase, but with a different time course from the PIK3CA mutation. Other patients had multiple mutations and showed consistent changes over time, such as patients 4956, 1653 and 6152, who had multiple mutations and showed consistent decreases for all observed mutations.

\section{Discussion}

Recent studies have shown that ESR1 mutations can be detected in metastatic ER + breast cancer tissues but generally not in primary tumour tissues collected before the initiation of endocrine therapy. Initial prevalence estimates varied widely, ranging from $12 \%(9 / 76)$ in an overall population of metastatic 
a
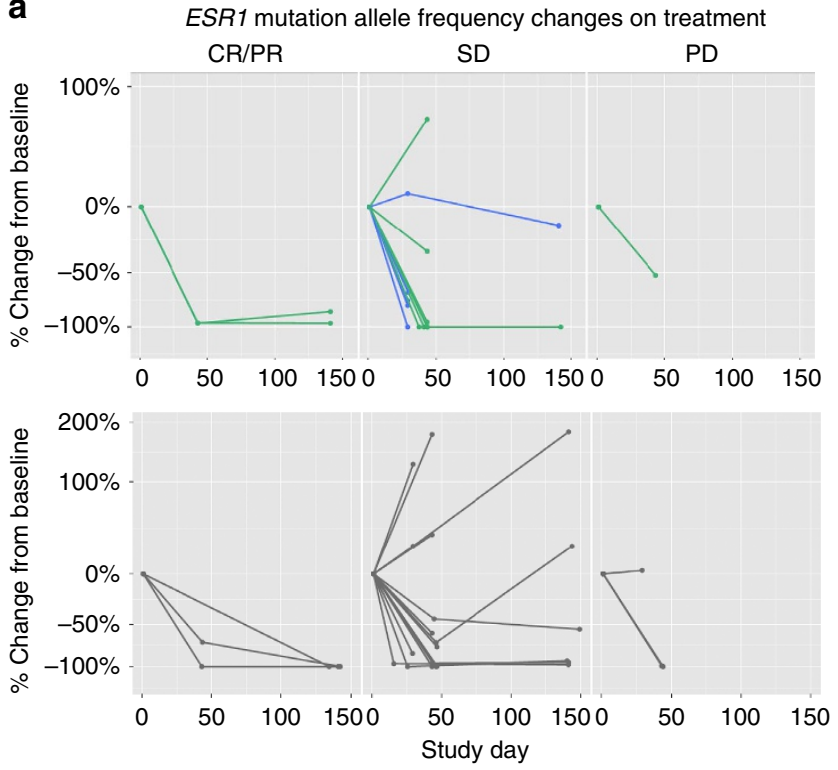

b
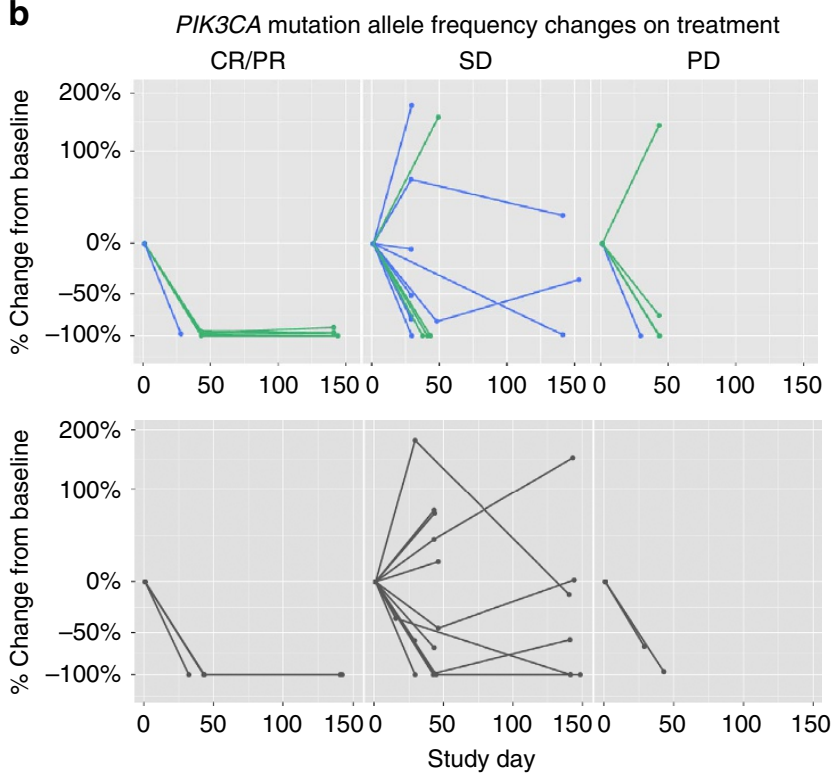

- $340 \mathrm{mg}$ pictilisib + FLV

- 260 mg pictilisib + FLV

Fulvestrant (FLV)

Figure 4 | Serial analysis of ctDNA mutation AF changes binned by best clinical response. (a) ESR1 and (b) PIK3CA ctDNA mutation AF changes while on part 1 (green) or part 2 (blue) pictilisib + fulvestrant or placebo + fulvestrant (grey) treatment arms. Patients are binned according to their best clinical response: CR/PR defined by RECIST criteria, and SD or $\mathrm{PD}$ as assessed by radiographic scans. Patients with a best response of non$\mathrm{CR} /$ non-PD for patients without measurable disease at baseline are binned with SD. Each line represents one patient with the per cent change of AF relative to baseline (study day 0 ) plotted for two or three time points. The mutation AF sum is plotted for patients with multiple ESR1 (a) or PIK3CA (b) mutations.

ER + breast cancers ${ }^{11}$ to $55 \%(6 / 11)$ in a small cohort of patients selected based on having had multiple rounds of hormonal therapy $^{12}$. These tissue-based findings have been extended to ctDNA in several small studies of limited numbers of patients, and recently in a cohort of 171 patients receiving AI therapy ${ }^{24}$. Guttery et al. ${ }^{19}$ analysed ctDNA from $48 \mathrm{ER}+$ breast cancer patients receiving a variety of therapies and showed that they could detect ESR1 mutations in 11 patients, while Sefrioui et al. ${ }^{20}$ analysed ctDNA and matched tissue from $7 \mathrm{ER}+$ patients and detected mutations in ctDNA in 4 of 6 patients with tumour mutations. Recently, Wang et al. ${ }^{25}$ showed that they could detect ESR1 mutations in 25\% of advanced breast cancer patients (9/29), and that changes in AF could be monitored longitudinally. Schiavon et al. ${ }^{24}$ recently showed convincingly that ESR1 mutations in ctDNA are rarely acquired during adjuvant $\mathrm{AI}$ treatment but are commonly selected by AI therapy for metastatic disease. To our knowledge, this report is the first to examine ESR1 mutations in a large, clinically well-defined cohort of patients who all received prior AI therapy, followed by the SERD fulvestrant in the context of a randomized trial.

These findings regarding the prevalence and overlap of PIK3CA and ESR1 help define an emerging model of polyclonal breast tumour evolution under the selective pressure of endocrine therapy. Genome-wide 'phylogenetic' analysis of breast cancer through next-generation sequencing has suggested that most PIK3CA mutations occur early in the process of tumour development and generally are associated with a tumour's dominant subclonal lineage ${ }^{26}$. In contrast, ESR1 mutations have been proposed to arise late and in a subclonal manner, since these mutations are generally not detected in primary tumour samples but only in metastatic lesions ${ }^{11,13,16}$. We found that PIK3CA mutations in ctDNA occurred on average at 10 -fold higher allelic frequencies than ESR1 mutations and generally each patient had a single mutation in PIK3CA. In contrast, ESR1 mutations were often polyclonal, as $40 \%$ of patients had more than one ESR1 mutation and in rare cases patients had four or five detectable low-frequency ESR1 mutations. Most patients in the study submitted only primary archival tissue for analysis, but a subset had metastatic biopsies available. Analysis of matched metastatic tumour tissue and ctDNA showed that while generally a single mutation could be detected in metastatic biopsies, matched ctDNA often showed additional mutations beyond the one identified in the tumour sample. These results suggest that mutational analysis of ctDNA may offer a superior platform to survey intrapatient mutational heterogeneity, likely by virtue of integrating data from diverse metastatic lesions, whereas analysis of one site may not provide an accurate picture of the overall genomic landscape.

Our data also provide a comprehensive survey of the prevalence of ESR1 mutations in advanced ER + metastatic breast cancer patients who have received prior AI therapy, as prior AI treatment was an enrollment criterion for the FERGI study. Overall, ESR1 mutations were detected in ctDNA from $37.3 \%$ of patients enrolled in part 1 of the clinical study. We found that ESR1 mutations showed a trend towards higher prevalence in patients with tumours classified as luminal A by PAM50 compared with luminal B (41.4 versus $31.8 \%$ ), as well as in patients with tumours harbouring PIK3CA mutations (44.4\%). ESR 1 mutations were also found in $44.4 \%$ of patients enrolled in the PIK3CA selected part of the FERGI study. These findings are consistent with a model wherein more oestrogen-dependent, luminal A tumours are particularly susceptible to selective pressure to maintain ER signalling in the presence of oestrogen depletion, and that acquisition of ESR1 mutations is a highly prevalent mechanism of resistance. Intriguingly, exploratory post hoc subgroup analysis in the FERGI study suggested improvement in PFS in patients with ER + and PgR + tumours treated with pictilisib plus fulvestrant (median of 7.2 months in the combination arm versus 3.7 months in the control arm $)^{17}$. The finding that ESR1 mutations are numerically enriched in luminal A, PIK3CA-mutated and $\mathrm{PgR}+$ tumours, together with the FERGI clinical results, may indicate that highly endocrine sensitive tumours are particularly sensitive to combined 
2310

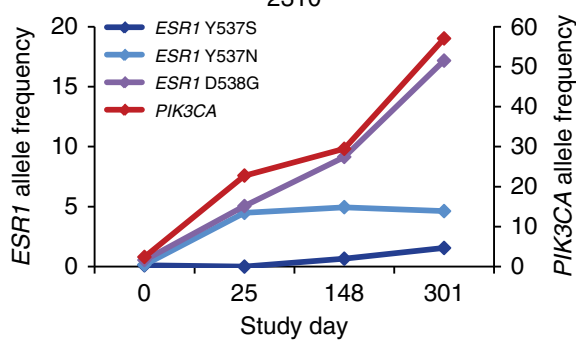

4251
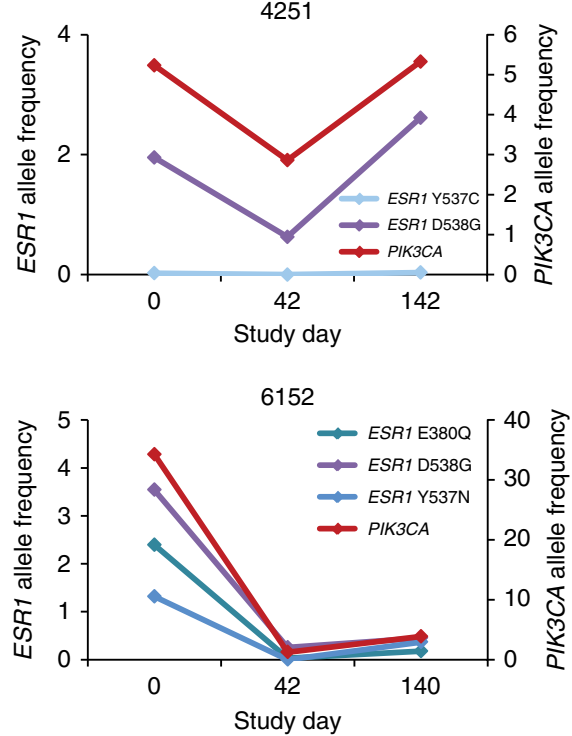

6951
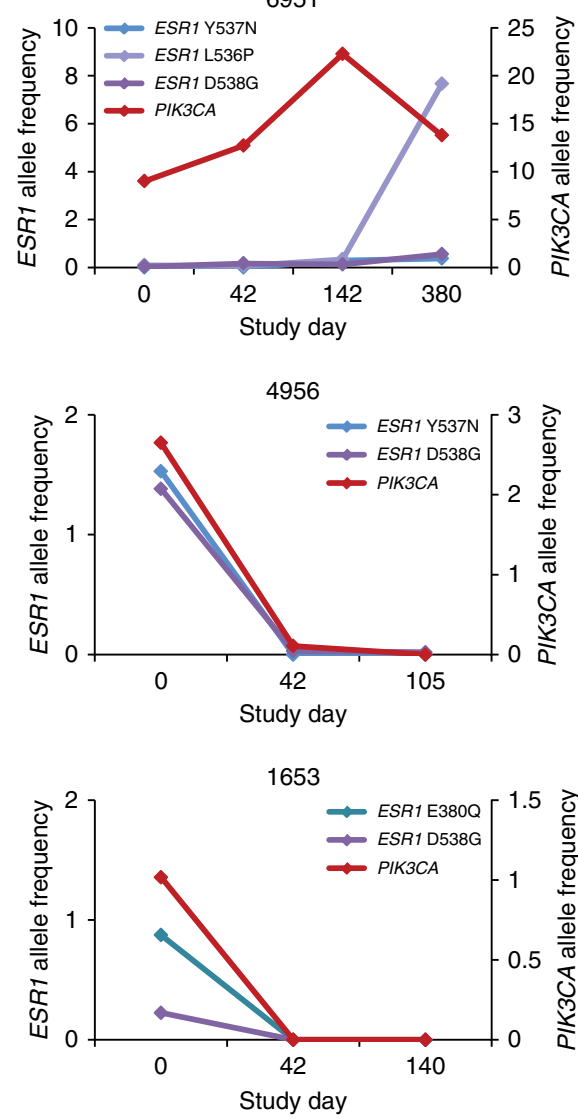

Figure 5 | Clonal heterogeneity of ESR1 mutations. Dynamic changes in ctDNA mutation AF changes for six patients while on treatment as examples of clonal heterogeneity of ESR1 mutations. Each line represents a different ESR1 or PIK3CA mutation, and raw AF values are plotted for each time point. Patients 2310 and 6951 demonstrate divergent behaviour of mutations. Patients 4956, 4251, 6152 and 1653 demonstrate consistent increases and/or decreases of $\mathrm{AF}$.

targeting of ER and PI3K signalling, a hypothesis that should be explored in future clinical studies.

The careful annotation of tumour collection date relative to AI therapy also allowed us to ask whether mutations were specifically associated with prior AI therapy. Indeed, we found that mutations were very infrequently detected in metastatic samples collected before AI therapy, but were common in patients where tissue was collected after the initiation of AI therapy, supporting the notion that these particular ESR1 mutations arise in the setting of AI exposure as a means of conferring ligandindependent oestrogen receptor signalling to tumour cells. These findings are consistent with recent work from Schiavon and colleagues, which suggested that ESR1 mutations are found exclusively in oestrogen receptor-positive breast cancer patients previously exposed to $\mathrm{AI}$, and that patients with ESR1 mutations had a substantially shorter PFS on subsequent AI-based therapy ${ }^{27}$. Overall, the data suggest a model of convergent evolution, wherein multiple metastatic subclones acquire independent activating mutations in ESR1 as a means of maintaining oestrogen signalling in the presence of anti-endocrine therapies. These data also have implications in terms of understanding the functional significance of the various hotspot mutations in ESR1. While the distribution of ESR1 mutations at the hotspot residues Y537 and D538 was consistent with previous reports, we found a slightly higher than expected distribution (26\% of patients with ESR1 mutations detected in plasma) of the N-terminal LBD mutation E380Q. While previous studies report increased sensitivity of this mutant to low concentrations of oestrogen and varying degrees of oestrogen-independent activity of this mutant in vitro in ER-negative models ${ }^{15,28}$, its functional significance in vivo remains unknown. We do not find significant ligand-independent activity of this mutant in ER-negative cells in vitro, consistent with studies from an ESR1 E380Q patient-derived xenograft mode ${ }^{15}$; however, our finding that the E380Q mutation occurred in two breast cancer patients' tissues and in one case at a high AF (18.1\%), and further, one of these samples were collected before the administration of AI therapy suggest a functional role of this mutation in ER+ breast cancer independent of AI resistance. Some clues to its mechanistic role come from a recent study showing that in contrast to the Y537S and D538G mutants, the ESR1 E380Q mutant cannot bind the $\mathrm{ER} \alpha$ co-regulatory protein prohibitin-2 (PHB2), which directly represses the transcriptional activity of activated $\mathrm{ER} \alpha^{29}$. These data suggest a possible mechanism whereby the E380Q mutant could enable increased ER signalling or prolonged ER signalling in the presence of oestrogen by virtue of its inability to bind the co-repressor PHB2, and our findings are consistent with this hypothesis.

Recent studies have highlighted the ability of mutational analysis of ctDNA to serve as a potential surrogate for antitumour activity and for therapeutic monitoring ${ }^{30}$. Dawson et al. ${ }^{31}$ used a next generation sequencing (NGS)-based approach to derive personalized panels of assays that could be used to monitor response over time and treatment, and found that circulating tumour DNA levels showed a greater dynamic range, and greater correlation with changes in tumour burden, than did CA 15-3 or 
circulating tumour cells, in advanced breast cancer patients. Similarly, Frenel et al. ${ }^{32}$ used serial next-generation sequencing of circulating ctDNA to monitor mutational response to a collection of targeted agents in patients from phase I studies, and found that the monitoring of mutation AF in consecutive plasma samples during treatment demonstrated potential treatment-associated clonal responses. Our results are broadly consistent with these studies but highlight some of the challenges associated with using ctDNA mutation status as a surrogate marker of antitumour activity. We found that patients that had radiographically confirmed RECIST responses (CR or PR) uniformly showed substantial decreases in both plasma PIK3CA and ESR1 AF, and that these changes were apparent at the earliest time point we assessed. However, we also observed plasma PIK3CA and ESR1 AF decreases of similar magnitude in a substantial fraction of patients whose best response was stable disease or progressive disease. This finding suggests a speculative model in which certain lesions in these patients were potentially responding to these therapies based on the decreased AFs, and possibly that progression was due to growth of other lesions that did not harbour the specific mutations being tracked. Analysis with multiplexed panels, perhaps based on NGS platforms, may be necessary to interrogate and monitor overall tumour status and response to therapy. In contrast, increases in plasma PIK3CA or ESR1 AFs were only seen in patients with stable disease or progressive disease, suggesting some potential utility in using the increase of mutation AFs to predict reduced clinical benefit if such findings can be confirmed in future prospective studies.

Our findings also have important implications for the treatment of patients with acquired ESR1 mutations. Previous studies have not addressed the impact of treatment with the SERD fulvestrant on PFS in patients with baseline ESR1 mutations. Our study does not provide any evidence that patients with plasma mutations in ESR1 have differential PFS with fulvestrant treatment, compared with patients without ESR1 mutations, suggesting ESR1 mutations in aggregate may not be associated with innate or acquired resistance to fulvestrant. Previous in vitro studies showing reduced sensitivity of the constitutively active mutants Y537S and D538G to fulvestrant suggested potential clinical resistance of ESR1 mutant patients to clinically achievable fulvestrant exposures ${ }^{11-13,33}$. These studies consistently showed that high suprapharmacologic concentrations of fulvestrant were required to inhibit transcriptional activity of $\mathrm{ER} \alpha$ mutants. Of note, these experiments were primarily conducted in ER-negative cell line models, and it is possible that $\mathrm{ER} \alpha$ mutants may behave differently on dimerization with WT ER $\alpha$ and in transcriptional complex with co-regulator proteins expressed in ER + breast cancers. Indeed, when these same mutations were expressed in two ER + cell lines, fulvestrant could downregulate WT and mutant ER $\alpha$ protein levels, and could partially suppress growth of the mutantexpressing cells ${ }^{15}$. Thus, the concentrations of fulvestrant needed to inhibit mutant $\mathrm{ER} \alpha$ activity may differ in the $\mathrm{ER}+$ versus ER - cell line setting, and effective clinical exposures of fulvestrant to address mutant $\mathrm{ER} \alpha$ may have been achieved in the FERGI study of ER + breast cancer patients, although the data presented were an exploratory post hoc analysis. Whether additional ESR1 mutations beyond the 12 evaluated in our study could alter the structure of $\mathrm{ER} \alpha$ and impair its degradation by fulvestrant, impacting therapeutic benefit from fulvestrant, remains to be determined. We also examined whether patients with more than one mutation, or a higher AF, potentially indicative of higher disease burden, showed longer PFS and again did not find any relationship between these parameters and clinical outcome. Moreover, serial analysis of mutations over time did not reveal the consistent appearance of ESR1 mutations at later time points, and in fact, ESR1 mutations in general showed a pattern of decreased AFs in most patients. This is in contrast to resistance mechanisms in other disease settings, such as KRAS mutations in patients treated with anti-EGFR antibodies, where a clear pattern of increases in KRAS in ctDNA occur post treatment and which are associated with disease progression ${ }^{21,34}$. Caution is required interpreting these findings since they are retrospective in nature and derived from exploratory subsets of a phase 2 trial, but our findings suggest that SERDs may have activity in patients with ESR1 mutations who are refractory to AI therapy and that prospective testing of this hypothesis is warranted. Nextgeneration orally available SERDs designed to more completely downregulate and degrade $\mathrm{ER} \alpha$ are currently in clinical development, and our findings can inform the design of future clinical trials involving fulvestrant as well as these next-generation SERDs $^{35,36}$. It will be of great interest to determine if these inhibitors are efficacious in patients harbouring multiclonal ESR1 mutations, ideally through prospective stratification with a validated diagnostic assay optimized for ctDNA.

\section{Methods}

Patient population. Study GDC4950g was an international, multicentre, randomized, double-blinded, placebo-controlled, phase 2 clinical trial evaluating the combination of pictilisib plus fulvestrant versus placebo plus fulvestrant in AI-resistant locally advanced or $\mathrm{MBC}^{37}$. The study was conducted in two parts. In part 1 , eligible patients were post-menopausal women aged $\geq 18$ years with $\mathrm{ER}+/$ HER2 - locally advanced or MBC appropriate for fulvestrant treatment based on national or local treatment guidelines, relapsed during or within 6 months of AI treatment in the adjuvant setting, or with PD during treatment with an $\mathrm{AI}$ in the metastatic setting. The most recent treatment before enrollment was required to be an $\mathrm{AI}$, with a minimum duration of 4 weeks of treatment before recurrence or PD. In part 1 of the study, 168 patients were randomized (1:1) to receive 28 -day cycles of fulvestrant $500 \mathrm{mg}$ on days 1 and 15 of cycle 1 and day 1 of subsequent cycles with either pictilisib $340 \mathrm{mg}$ QD $(n=89)$ or matching placebo $(n=79)$. Part 1 also enrolled 32 patients to receive the PI3K/mTOR inhibitor GDC-0980, but this arm was discontinued based on safety data and the recommendation of the Internal Monitoring Committee. The primary analysis was based on a 6-month median duration follow-up. Inclusion criteria for part 2 were similar to those for part 1 , except that in part 2 all patients were required to have a PIK3CA-mutated tumour and the requirement for an AI to be the last therapy was removed. Part 2 randomized (2:1) 61 patients to receive fulvestrant with either pictilisib $260 \mathrm{mg}$ QD $(n=41)$ or placebo $(n=20)$. The study was conducted in accordance with Good Clinical Practice guidelines and the Declaration of Helsinki. Written informed consent was obtained from all patients before enrollment, in agreement with approved protocols from respective ethics committees at every site.

Patient plasma and tissue samples. Baseline plasma samples for ctDNA analysis were collected from 156 of the 168 randomized part 1 patients. ctDNA was tested for PIK3CA mutations in all 156 samples, of which 142 had matched PIK3CA mutation status from tumour tissue for specificity and sensitivity analysis. ctDNA was tested for ESR1 mutations from 153 of 156 patients, and an additional 24 patients from the GDC-0980 arm. ctDNA was also tested for PIK3CA and ESR1 mutations from 60 and 54 part 2 patients, respectively. PIK3CA and ESR 1 ctDNA prevalence and associations with patient demographics and molecular characteristics were based on the 156 or 153 part 1 patients, respectively. Residual plasma samples from pharmacokinetic analysis were used for ctDNA analysis from 71 patients ( 49 from part 1 and 22 from part 2) that consented to the use of the residual material for exploratory biomarker analyses. These samples were collected at cycle 2 day 15 (part 1 ) or cycle 2 day 1 (part 2), cycle 6 day 1 and crossover cycle 2 day 15 (for patients that received crossover therapy). Tumour tissue was tested for ESR1 mutations from a total of 102 patients, 64 and 27 from part 1 and part 2, respectively, and an additional 11 patients from the GDC-0980 arm. Patient tissue samples were chosen based on the presence of an ESR1 mutation in the baseline plasma sample or if the tissue was collected after the patient was diagnosed with metastatic disease. Two tissue samples tested for ESR1 mutations were from fresh biopsies collected within 3 weeks of the blood collection, and the remaining tissue samples were archival.

Tissue mutation testing. Genomic DNA was isolated from formalin-fixed paraffin-embedded tumour tissue and tested for PIK3CA mutation status by quantitative real-time PCR for activating missense mutations C420R, E542K, E545A/G/K and $\mathrm{H} 1047 \mathrm{~L} / \mathrm{R} / \mathrm{Y}$ at a central laboratory. Droplet digital PCR probe assays were designed to assess ESR1 mutation status for 10 mutations: E380Q (1508 G > C); P535H (1974 C > A); L536H (1977T > A); L536P (1977T > C); L536Q (1977-8 TC $>$ AG); L536R $(1977$ T $>$ G); Y537C $(1980 A>G) ;$ Y 537N $(1979 \mathrm{~T}>A) ;$ Y $537 S$ 
(1980A > C); and D538G (1983A > G). For patient samples with sufficient DNA available $(n=60)$, all mutations detected in plasma (excluding ESR1 S463P) were assessed in the matched tissue. For patients with limiting DNA available $(n=15)$, only the one or two mutations with the highest allele fractions detected in plasma were assessed in tissue. For patient samples with no mutation detected in plasma, all mutations were assessed in matched tissue samples if there was sufficient DNA $(n=17)$ or only the most common mutations (Y537C/N/S, D538G and E380Q) if there was limited DNA $(n=10)$. An ESR1 WT assay containing a $5^{\prime}$ HEX-labelled probe was used as a reference to estimate WT copy number and to calculate mutant $\mathrm{AF}$ in each individual sample. ESR1 mutant assays containing a $5^{\prime} \mathrm{FAM}-$ labelled probe were designed to use the identical forward and reverse primer sequences as the WT assays and to differ only in the probe sequences. ESR1 assay sequences are listed in Supplementary Table 7. All assays were performed on the QX200 Droplet Digital PCR System (BioRad) according to the manufacturer's protocol and using an annealing temperature of $55^{\circ} \mathrm{C}$. Each assay run included ESR1 WT and mutant oligonucleotide controls and a human WT genomic DNA control (Roche) to determine fluorescence amplitude thresholds for positive and negative mutation calls. To achieve a sensitivity level of $\sim 0.02 \%$ mutant AF per individual ESR1 mutation, $20 \mathrm{ng}$ genomic DNA was assessed per mutation. ESR1 mutant AFs were calculated using QuantaSoft software (BioRad).

Plasma mutation testing. ctDNA was tested for PIK3CA and ESR1 mutations using the OncoBEAM BC1 BEAMing Digital PCR panel (conducted at Sysmex, Hamburg, Germany), as previously described ${ }^{38}$. Briefly, DNA (3-1,500 ng) was isolated from 1 to $2 \mathrm{ml}$ of plasma and pre-amplified in a PCR reaction using gene specific primers with a tag sequence. The pre-amplified DNA was then subjected to an emulsion PCR containing tag specific primers bound to magnetic beads. The resulting beads with bound amplified DNA were hybridized to WT or mutant specific fluorescent probes followed by flow cytometry to quantitate the fraction of mutant alleles to total DNA alleles. The assay detects 9 PIK3CA mutations $(\mathrm{C} 420 \mathrm{R}$, E542K, E545K/G, Q546K, M1043I and H1047R/L/Y) and 12 ESR1 mutations (E380Q, S463P, V524E, P535H, L536H/P/Q/R, Y537C/N/S and D538G), and has a limit of detection of $0.02 \%$ assuming the DNA input is at least $16 \mathrm{ng}$. Approximately $55 \%$ of all plasma samples tested had sufficient DNA for this limit of detection, and $97 \%$ of the samples had sufficient DNA to detect $0.06 \%$. Unless otherwise noted, all reported mutant allele fractions are a composite of all ESR1 or PIK3CA mutant allele fractions for a given sample, if the sample has two or more ESR1 or PIK3CA mutations detected.

Gene expression. Haematoxylin-eosin sections were prepared for all samples and were reviewed by a pathologist to confirm diagnosis and assess tumour content. RNA extraction and gene expression analysis was performed as previously described ${ }^{39}$. Briefly, FFPE sections were macrodissected to enrich for neoplastic tissue followed by RNA extraction using the High Pure FFPE RNA Micro Kit (Roche Applied Sciences). Gene expression was subsequently determined using the NanoString nCounter Analysis System on a custom gene panel which includes 50 genes from the PAM50 breast cancer intrinsic classification signature ${ }^{22}$, listed in Supplementary Table 8 . A total of $200 \mathrm{ng}$ of RNA was hybridized to the codeset overnight at $65^{\circ} \mathrm{C}$ according to the NanoString protocol. Samples were loaded onto the NanoString nCounter Prep Station and read using the NanoString nCounter Digital Analyzer. Raw expression data was then $\log 2$-transformed and normalized against included housekeeping genes.

Breast cancer molecular classification. PAM50 subtype prediction was carried out using a random-forest-based classifier that was derived from an independent training set of 157 breast cancer samples (data not shown) and 50 genes from the PAM50 signature 22 . We assigned PAM50 subtypes for the training samples based on consensus calls from both public nearest-centroid-based PAM50 classification and hierarchical clustering approaches to reduce platform and population biases. Among them, 112 samples had consistent calls from both approaches and formed the final training set. A random-forest-based classifier was then developed with an estimated out-of-bag error rate of $7.1 \%$ and applied to predict new samples.

Data availability. The authors declare that the data supporting the findings of this study are available within the article and its Supplementary Information files.

\section{References}

1. Clark, G. M., Osborne, C. K. \& McGuire, W. L. Correlations between estrogen receptor, progesterone receptor, and patient characteristics in human breast cancer. J. Clin. Oncol. 2, 1102-1109 (1984).

2. Ariazi, E. A., Ariazi, J. L., Cordera, F. \& Jordan, V. C. Estrogen receptors as therapeutic targets in breast cancer. Curr. Top. Med. Chem. 6, 181-202 (2006).

3. Osborne, C. K. Tamoxifen in the treatment of breast cancer. N. Engl. J. Med. 339, 1609-1618 (1998).

4. Burstein, H. J. et al. Adjuvant endocrine therapy for women with hormone receptor-positive breast cancer: american society of clinical oncology clinical practice guideline focused update. J. Clin. Oncol. 32, 2255-2269 (2014).
5. Nardone, A., De Angelis, C., Trivedi, M. V., Osborne, C. K. \& Schiff, R. The changing role of ER in endocrine resistance. Breast 24, S60-S66 (2015).

6. Robertson, J. F. et al. Fulvestrant versus anastrozole for the treatment of advanced breast carcinoma in postmenopausal women: a prospective combined analysis of two multicenter trials. Cancer 98, 229-238 (2003).

7. Musgrove, E. A. \& Sutherland, R. L. Biological determinants of endocrine resistance in breast cancer. Nat. Rev. Cancer 9, 631-643 (2009).

8. Johnston, S. R. et al. Comparison of estrogen receptor DNA binding in untreated and acquired antiestrogen-resistant human breast tumors. Cancer Res. 57, 3723-3727 (1997).

9. Dodwell, D., Wardley, A. \& Johnston, S. Postmenopausal advanced breast cancer: options for therapy after tamoxifen and aromatase inhibitors. Breast 15 , 584-594 (2006).

10. Zhang, Q. X., Borg, A., Wolf, D. M., Oesterreich, S. \& Fuqua, S. A. An estrogen receptor mutant with strong hormone-independent activity from a metastatic breast cancer. Cancer Res. 57, 1244-1249 (1997).

11. Jeselsohn, R. et al. Emergence of constitutively active estrogen receptor-alpha mutations in pretreated advanced estrogen receptor-positive breast cancer. Clin. Cancer Res. 20, 1757-1767 (2014).

12. Robinson, D. R. et al. Activating ESR1 mutations in hormone-resistant metastatic breast cancer. Nat. Genet. 45, 1446-1451 (2013).

13. Toy, W. et al. ESR1 ligand-binding domain mutations in hormone-resistant breast cancer. Nat. Genet. 45, 1439-1445 (2013).

14. Carlson, K. E., Choi, I., Gee, A., Katzenellenbogen, B. S. \& Katzenellenbogen, J. A. Altered ligand binding properties and enhanced stability of a constitutively active estrogen receptor: evidence that an open pocket conformation is required for ligand interaction. Biochemistry 36, 14897-14905 (1997).

15. Li, S. et al. Endocrine-therapy-resistant ESR1 variants revealed by genomic characterization of breast-cancer-derived xenografts. Cell Rep. 4, 1116-1130 (2013).

16. Oesterreich, S. \& Davidson, N. E. The search for ESR1 mutations in breast cancer. Nat. Genet. 45, 1415-1416 (2013).

17. Krop, I. E. et al. Phase 2 randomised study of the PI3-kinase inhibitor pictilisib plus fulvestrant versus placebo plus fulvestrant in patients with oestrogen receptor-positive, aromatase inhibitor-resistant advanced or metastatic breast cancer: the FERGI trial. Lancet Oncol. (in the press).

18. Chu, D. et al. ESR1 mutations in circulating plasma tumor DNA from metastatic breast cancer patients. Clin. Cancer Res. 22, 993-999 (2015).

19. Guttery, D. S. et al. Noninvasive detection of activating estrogen receptor 1 (ESR1) mutations in estrogen receptor-positive metastatic breast cancer. Clin. Chem. 61, 974-982 (2015).

20. Sefrioui, D. et al. Short report: monitoring ESR1 mutations by circulating tumor DNA in aromatase inhibitor resistant metastatic breast cancer. Int. J. Cancer 137, 2513-2519 (2015).

21. Diaz, Jr L. A. et al. The molecular evolution of acquired resistance to targeted EGFR blockade in colorectal cancers. Nature 486, 537-540 (2012).

22. Parker, J. S. et al. Supervised risk predictor of breast cancer based on intrinsic subtypes. J. Clin. Oncol. 27, 1160-1167 (2009).

23. Ascierto, P. A. et al. Phase II trial (BREAK-2) of the BRAF inhibitor dabrafenib (GSK2118436) in patients with metastatic melanoma. J. Clin. Oncol. 31, 3205-3211 (2013).

24. Schiavon, G. et al. Analysis of ESR1 mutation in circulating tumor DNA demonstrates evolution during therapy for metastatic breast cancer. Sci. Transl. Med. 7, 313ra182 (2015).

25. Wang, P. et al. Sensitive detection of mono- and polyclonal ESR1 mutations in primary tumors, metastatic lesions, and cell-free DNA of breast cancer patients. Clin. Cancer Res. 22, 1130-1137 (2016).

26. Nik-Zainal, S. et al. The life history of 21 breast cancers. Cell 149, 994-1007 (2012).

27. Garcia-Murillas, I. et al. Mutation tracking in circulating tumor DNA predicts relapse in early breast cancer. Sci. Transl. Med. 7, 302ra133 (2015).

28. Potthoff, S. J., Romine, L. E. \& Nardulli, A. M. Effects of wild type and mutant estrogen receptors on DNA flexibility, DNA bending, and transcription activation. Mol. Endocrinol. 10, 1095-1106 (1996).

29. Chigira, T., Nagatoishi, S. \& Tsumoto, K. Differential binding of prohibitin-2 to estrogen receptor alpha and to drug-resistant ERalpha mutants. Biochem. Biophys. Res. Commun. 463, 726-731 (2015).

30. De Mattos-Arruda, L. et al. Circulating tumour cells and cell-free DNA as tools for managing breast cancer. Nat. Rev. Clin. Oncol. 10, 377-389 (2013).

31. Dawson, S. J., Rosenfeld, N. \& Caldas, C. Circulating tumor DNA to monitor metastatic breast cancer. N. Engl. J. Med. 369, 93-94 (2013).

32. Frenel, J. S. et al. Serial next-generation sequencing of circulating cell-free DNA evaluating tumor clone response to molecularly targeted drug administration. Clin. Cancer Res. 21, 4586-4596 (2015).

33. Merenbakh-Lamin, K. et al. D538G mutation in estrogen receptor-alpha: a novel mechanism for acquired endocrine resistance in breast cancer. Cancer Res. 73, 6856-6864 (2013).

34. Misale, S. et al. Emergence of KRAS mutations and acquired resistance to antiEGFR therapy in colorectal cancer. Nature 486, 532-536 (2012). 
35. De Savi, C. et al. Optimization of a novel binding motif to (E)-3-(3,5-Difluoro4-((1R,3R)-2-(2-fluoro-2-methylpropyl)-3-methyl-2,3,4,9-tetra hydro-1Hpyrido[3,4-b]indol-1-yl)phenyl)acrylic Acid (AZD9496), a potent and orally bioavailable selective estrogen receptor downregulator and antagonist. J. Med. Chem. 58, 8128-8140 (2015).

36. Lai, A. et al. Identification of GDC-0810 (ARN-810), an orally bioavailable selective estrogen receptor degrader (SERD) that demonstrates robust activity in tamoxifenresistant breast cancer xenografts. J. Med. Chem. 58, 4888-4904 (2015).

37. Krop, I. et al. The FERGI phase II study of the PI3K inhibitor pictilisib (GDC0941) plus fulvestrant vs fulvestrant plus placebo in patients with ER +, aromatase inhibitor (AI)-resistant advanced or metastatic breast cancer-part I results. Cancer Res. 75, S2-02 (2015).

38. Diehl, F. et al. Circulating mutant DNA to assess tumor dynamics. Nat. Med. 14, 985-990 (2008).

39. Wilson, T. R. et al. Development of a robust RNA-based classifier to accurately determine ER, PR, and HER2 status in breast cancer clinical samples. Breast Cancer Res. Treat. 148, 315-325 (2014).

\section{Acknowledgements}

We thank the patients, their families and the investigators who participated in FERGI study. The authors are grateful to the National Institute for Health Research and Cancer Research UK for funding Barts/Brighton Experimental Cancer Medicine Centre in support of the FERGI clinical trial. In addition, we thank Lori Friedman, Jeff Hager and Ciara Metcalfe for helpful discussions, and Joseph Ware for kindly providing access to plasma samples.

\section{Author contributions}

J.M.S., K.W., S.G. and M.R.L. conceived of and designed the study; T.R.W., J.M.S. and K.W. developed methodology; K.W. and H.S. performed laboratory experiments; J.Q. and M.C. conducted statistical analyses; J.A. provided oversight for outsourced studies; J.M.S., S.G., K.W., J.Q., T.R.W., H.S., J.A., M.K.D., M.C., I.T.C., L.C.A., G.M.H., S.J., I.K., P.S. and M.R.L. analysed and interpreted data; J.M.S., K.W. and M.R.L. wrote the manuscript with input and final approval from all authors.

\section{Additional information}

Supplementary Information accompanies this paper at http://www.nature.com/ naturecommunications

Competing financial interests: J.M.S., S.G., K.W., J.Q., T.R.W., H.S., J.A., M.K.D., M.C., I.T.C., L.C.A., G.M.H. and M.R.L. were all employed by Genentech, Inc during the time the study was done. I.K., S.J. and P.S. were investigators on the FERGI clinical study and received research funding from Genentech.

Reprints and permission information is available online at http://npg.nature.com/ reprintsandpermissions/

How to cite this article: Spoerke, J. M. et al. Heterogeneity and clinical significance of ESR1 mutations in ER-positive metastatic breast cancer patients receiving fulvestrant. Nat. Commun. 7:11579 doi: 10.1038/ncomms11579 (2016).

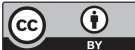

This work is licensed under a Creative Commons Attribution 4.0 International License. The images or other third party material in this article are included in the article's Creative Commons license, unless indicated otherwise in the credit line; if the material is not included under the Creative Commons license, users will need to obtain permission from the license holder to reproduce the material. To view a copy of this license, visit http://creativecommons.org/licenses/by/4.0/ 